\title{
BAYESIAN INFERENCE IN CREDIBILITY THEORY
}

\author{
L. D'Hooge and M. J. Goovaerts \\ Leuven (K.U.L.)
}

\begin{abstract}
In I967, Bühlmann has shown that the credibility formula was the best linearized approximation to the exact Bayesian forecast.

His result for the credibility factor $z=V_{\theta} E(\xi / \theta) / V\left(\mathbf{I} / n \sum_{t=1}^{n} \xi_{t}\right)$ can be found back by means of some Bayesian inference techniques. Introducing a uniform prior probability density function for the credibility factor provides us with a method for estimating $z$, a correction term to the Bühlmann's result is obtained. It is shown how prior boundary conditions can be introduced.
\end{abstract}

\section{INTRODUCTION}

In the present contribution the credibility factor $z$ will be introduced by means of some adequate Bayesian inference technique. As has already been remarked several times [I] completely different methods leading to the same expression for $z$. We will show that also in a Bayesian framework for estimating parameters the same expressions can be obtained under certain general conditions. However we aim to suggest a method for deriving $z$ as a function, being the mean value of a stochastical variable $z$ imposing some inequality constraints. Let us first recall some elements of Bayesian inference [2]. Let $f_{X}(x, \lambda)$ be a notation for the distribution density function of a one dimensional stochastical variable $X$. This distribution depends on the parameter $\lambda$. Of course the mathematical admissable range of $\Lambda$, say $\lambda \in \Lambda$, can be determined by examining the given function $f_{X}(x, \lambda) . \Lambda$ is supposed to be a continuous parameter space. It is clear that for a Gaussian distribution density:

$$
f_{X}(x, \lambda)=\frac{\mathrm{I}}{\sqrt{2 \pi} \sigma} \exp \left\{-(x-\lambda)^{2} / 2 \sigma^{2}\right\}
$$

where $\sigma$ is a number, $\Lambda$ can be defined by the inequality: $\Lambda:\{\lambda \mid$ $-\infty<\lambda<+\infty\}$. In fact $f_{X}(x, \lambda)$ can be interpreted as the distribution density of $X$, under the condition that $\lambda$ is given, it is 
$f_{X}(x, \lambda)$ is the joint posterior distribution density of $X$ with given $\lambda$. In estimating $\lambda$ in a Bayesian way one ought to consider the joint distribution density of $\lambda$, given $\left(x_{1}, \ldots, x_{n}\right)$, where $\left(x_{1}, \ldots, x_{n}\right)$ is constructed from some experimental data. The question that arises is what prior density of $\lambda$ has to be taken, anyhow the economical meaning of the parameter itself often determines this prior density and certainly the range of the stochastical variable $X$. In case of "knowing nothing" [2] Jeffreys' rule becomes:

"If $\lambda \in[-\infty,+\infty]$ then the prior density $\Phi_{\lambda}(\lambda)=c$, which is an improper density; if $\lambda \in[0,+\infty]$ then one has $\Phi_{\lambda}(\lambda)=c / \lambda$ ".

Given a vector $\bar{x}^{0}\left(x_{1}^{0}, \ldots, x_{n}^{0}\right)$ of $n$ independent observations the likelihood-function of the sample is given by:

$$
L\left(\bar{x}^{0} / \lambda\right)=\prod_{j=1}^{n} f_{X}\left(x_{j}^{0}, \lambda\right)
$$

and the Bayesian estimator of $\lambda$ is known to be given by:

$$
\text { B.E. }(\lambda)=\frac{\int_{\Lambda} d \lambda L\left(\bar{x}^{0} / \lambda\right) \Phi_{\lambda}(\lambda) \cdot \lambda}{\int_{\Lambda} d \lambda L(\bar{x} / \lambda) \Phi_{\lambda}(\lambda)}
$$

It can be shown that under some general conditions [3], B.E.( $(\lambda)$ can also be obtained by the least square method. Let us introduce next some notations for describing the credibility model.

A collective of heterogenous risks, in which each member is characterized by a risk parameter $\theta$ is considered. The claim experience for a certain time period $t$ is a random variable with known distribution:

$$
P_{t}(x / \theta)=\operatorname{Prob}\left(\xi_{t} \leq x / \theta\right) \quad(t=\mathrm{I}, 2, \ldots)
$$

and with density $p_{t}(x / \theta)$.

We will assume the $\xi_{t}$ to be mutually independent.

If the individual $\theta$ were not known a prior distribution $U(\theta)$ is introduced. As is explained f.e. in [4] the forecast density of the next year's risk would be the conditional density:

$$
P_{n+1}\left(y / x_{1}, \ldots, x_{n}\right)=\frac{\int P_{n+1}(y / \theta) \prod_{t-1}^{n} P_{t}\left(x_{t} / \theta\right) d U(\theta)}{\int \prod_{t=1}^{n} P_{t}\left(x_{t} / \theta\right) d U(\theta)}
$$


The fair premium for the year $n+\mathbf{I}$ would then be:

$E\left(\xi_{n+1} / \xi_{t}=x_{t}(t=\mathrm{I}, 2, \ldots, n)\right)=\int y P_{n+1}\left(y / x_{1}, x_{2}, \ldots, x_{n}\right) d y$

The collective distribution of the premium is given by:

$$
P_{t}(x)=E_{\theta}\left(P_{t}(x / \theta)\right)=\int P_{t}(x / \theta) d U(\theta)
$$

The premium, not taking into account the individual experience data, would be given by:

$$
E\left(\xi_{n+1}\right)=\int x P_{n+1}(x) d x=E\left(\xi_{n}\right)=\ldots=E\left(\xi_{1}\right)
$$

with :

$$
P_{t}(x)=P_{t^{\prime}}(x) \text { for } \quad \forall t, t^{\prime}
$$

\section{The Approximate Credibility Formula}

The fair premium for the year $n+\mathrm{I}$ is usually written as a linear combination of the collective mean $E(\xi)$ and the sample mean $(I / n) \sum_{i=1}^{n} x_{t}$ of the individual experience data

$$
E\left(\xi_{n+1} / \bar{x}\right) \cong(\mathrm{I}-z) E(\xi)+z \frac{\mathrm{I}}{n} \sum_{t=1}^{n} x_{t}
$$

The factor $z$ is called the credibility factor, and was assumed to be of the form:

$$
z=\frac{n}{n+N}
$$

Bühlmann showed that the best approximation to $E\left(\xi_{n+1} / \bar{x}\right)$ in the sense of minimizing [5] [6]

$$
I=E\left\{\left[E\left(\xi_{n+1} 1 \bar{x}\right)-a-b \frac{I}{n} \sum_{i=1}^{n} \xi_{t}\right]^{2}\right\}
$$

is given by:

$$
\begin{aligned}
& a=(\mathrm{I}-b) E(\xi) \\
& b=\frac{V_{\theta} E\left(\xi_{n+1} / \theta\right)}{V\left(\frac{\mathrm{I}}{n} \sum_{i=1}^{n} \xi_{t}\right)}
\end{aligned}
$$


If course one can also look for the best approximation to $E\left(\xi_{n+1} / \bar{x}\right)$ in the sense of minimizing:

$$
I=E\left\{\left[E\left(\xi_{n+1} \mid \bar{x}\right)-(\mathrm{I}-b) E(\xi)-b \frac{\mathrm{I}}{n} \sum_{t=1}^{n} \xi_{t}\right]^{2}\right\}
$$

The same result is obtained as in the previous model. form:

The credibility factor $b=\frac{V_{\theta} E\left(\xi_{n+1} / \theta\right)}{V\left(\frac{\mathrm{I}}{n} \sum_{t=1}^{n} \xi_{t}\right)}$ can still be cast into the

$$
b=\frac{n}{n+N}
$$

with

$$
N=\frac{E_{\theta} V(\xi / \theta)}{V_{\theta} E(\xi / \theta)}
$$

The same result can still be obtained introducing Bayesian inference techniques. Indeed, in the present case the likelihood function becomes:

$$
L(b)=c e^{-1 / 2 E\left\{\left[E\left(\xi_{n+1} / \bar{x}\right)-E(\xi)-b\left(1 / n \sum_{t=1}^{n} \xi_{l}-E(\xi)\right)\right]^{2}\right\}}
$$

Because no information of $E\left(\xi_{n+1} / \bar{x}\right)$ is available in the sense that an experiment would give us some values for $E\left(\xi_{n+1} / \xi_{n}, \ldots, \xi_{1}\right)$, the usual summation over the number of experiments becomes the operator $E$, where the integrations have to be carried out over the $(n+\mathrm{I})$-dimensional space generated by all prior possible $\left\{\xi_{\mathrm{I}}, \xi_{2}\right.$, $\left.\ldots, \xi_{n} ; \theta\right\}$ with measure

$$
d U(\theta) \prod_{t=1}^{n} p\left(x_{t} / \theta\right) d x_{t}
$$

Following Jeffreys the prior distribution density turns out to be a constant. So the posterior distribution function $p d f(b)$ turns out to be:

$$
p d f(b) \cong e^{-1 / 2 E\left[\left[E\left(\xi_{n+1} / \bar{x}\right)-E(\xi)-b\left(1 / n \sum_{i=1}^{n} \xi_{t}-E(\xi)\right)\right]^{2}\right\}}
$$


The Bayesian estimation of $b$ turns out to be:

$$
\text { B.E. }(b)=\frac{\int_{-\infty}^{+\infty} b d b e^{-1 / 2 E\left\{\left[E\left(\xi_{n+1} / \bar{x}\right)-E(\xi)-b\left(1 / n \sum_{t=1}^{n} \xi_{t}-E(\xi)\right)\right]^{2}\right\}}}{\int_{-\infty}^{+\infty} d b e^{-1 / 2 E\left\{\left[E\left(\xi_{n+1} / \bar{x}\right)-E(\xi)-b\left(1 / n \sum_{t=1}^{n} \xi_{t}-E(\xi)\right)\right]^{2}\right\}}}
$$

The Gaussian integrals in the nominator and in the denominator are readely performed:

$$
z(t)=\text { B. E. }(b)=\frac{V_{\theta} E\left(\xi_{n+1} / \theta\right)}{V\left(\frac{\mathrm{I}}{n} \sum_{i=1}^{n} \xi_{t}\right)}
$$

3. The Approximate Credibility Formula, Introducing

\section{UNIFORM PRIORS}

It is clear that the nature of the approximate formula has as a consequence:

$$
0<z(t)<\mathrm{I}
$$

To take into account these constraints our $p d f(b)$ is constructed as a product of the likelihood function with the uniform prior $p(b)$ defined as:

$$
p(b)=\left\{\begin{array}{lll}
0 & \text { if } & b<0 \\
\mathrm{I} & \text { if } & 0 \leq b \leq \mathrm{I} \\
\mathrm{O} & \text { if } & \mathrm{I}<b
\end{array}\right.
$$

So the Bayesian estimator of the credibility factor is given by:

$$
z=\frac{\int_{0}^{1} b d b \exp \left\{-\frac{1}{2} E\left\{\left[E\left(\xi_{n+1} / \bar{x}\right)-E(\xi)-b\left(\frac{1}{n} \sum_{t-1}^{n} \xi_{t}-E(\xi)\right)\right]^{2}\right\}\right.}{\int_{0}^{1} d \exp \left\{-\frac{1}{2} E\left\{\left[E\left(\xi_{n+1} / \bar{x}\right)-E(\xi)-b\left(\frac{1}{n} \sum_{t-1}^{n} \xi_{t}-E(\xi)\right)\right]^{2}\right\}\right.}
$$

which can still be cast into the form:

$$
z=\frac{\int_{0}^{1} e^{-1 / 2 b^{2} V\left(1 / n \sum_{t=1}^{n} \xi_{t}\right)+b V_{\theta} E\left(\xi_{n+1} / \theta\right)} b d b}{\int_{0}^{1} e^{-1 / 2 b^{2} V\left(1 / n \sum_{t=1}^{n} \xi_{t}\right)+b V_{\theta} E\left(\xi_{n+1} / \theta\right)} d b}
$$


Making use of a result obtained by H. Bühlmann [6]

$$
V\left(\frac{\mathrm{I}}{n} \sum_{i=1}^{n} \xi_{t}\right)=\left(\mathrm{I}-\frac{\mathrm{I}}{n}\right) V_{\theta} E(\xi / \theta)+\frac{V(\xi)}{n}=V_{\theta} E(\xi / \theta)+E_{\theta} V(\xi / \theta) \cdot \frac{\mathrm{I}}{n}
$$

one is faced with:

$$
z(n)=\frac{\int_{0}^{1} b d b \exp \left\{-\frac{1}{2} b^{2}\left[\left(\mathrm{I}-\frac{\mathrm{I}}{n}\right) V_{\theta} E(\xi / \theta)+\frac{V(\xi)}{n}\right]+b V_{\theta} E(\xi / \theta)\right\}}{\int_{0}^{1} d b \exp \left\{-\frac{1}{2} b^{2}\left[\left(\mathrm{I}-\frac{\mathrm{I}}{n}\right) V_{\theta} E(\xi / \theta)+\frac{V(\xi)}{n}\right]+b V_{\theta} E(\xi / \theta)\right\}}
$$

By means of one partial integration one obtains:

$$
\begin{aligned}
& z(n)=\frac{n}{n+\frac{E_{\theta} V(\xi / \theta)}{V_{\theta} E(\xi / \theta)}-} \\
& \frac{\exp \left\{\frac{1}{2} V_{\theta} E(\xi / \theta)-\frac{\mathrm{I}}{2 n} E_{\theta} V(\xi / \theta)\right\}-\mathrm{I}}{\left[V_{\theta} E(\xi / \theta)+E_{\theta} V(\xi / \theta) \frac{\mathrm{I}}{n}\right] \int_{0}^{1} e^{-1 / 2 b^{2}\left[V_{\theta} E(\xi / \theta)+E_{\theta} V(\xi / \theta) 1 / n\right]+b V_{\theta} E\left(\xi_{n+1} / \theta\right)} d b}
\end{aligned}
$$

So if one neglects the correction term the result of (4) Bühlmann $[6]$ is found back.

Of course it is possible to think about other prior densities for $b$, depending on $n$. In fact there is no mathematical argument taking an arbitrary function $z(t)$. Indeed:

$$
E\left[E\left(\xi_{n+1} / \theta, \bar{x}\right)-(\mathrm{I}-z(t)) E(\xi)-z(t) \frac{\mathrm{I}}{n} \sum_{t=1}^{n} \xi_{t}\right]=0
$$

for all $z(t)$.

4. The Approximate Credibility Formula, Introducing a

\section{Combination of Normal Priors}

It is clear that in our expression for $z(t)$ the limit of the correction term for $n \rightarrow \infty$ doesn't approach to zero. To avoid this difficulty, if it is one, other prior densities can be introduced. 
Indeed, some conditions like

$$
z(0)=0 ; \quad z(\infty)=\mathrm{I}
$$

can be introduced. It is sufficient to take $\phi_{b}(b)$ defined by:

$$
\phi_{b}(b)=\exp \left\{-(b-\mathrm{I})^{2} \frac{n}{2}-\frac{b^{2}}{2 n}\right\} \quad N_{b}:[\mathrm{O}, \mathrm{I}]
$$

Indeed:

$$
\lim _{n \rightarrow \infty} \phi_{b}(b)=\delta(b-\mathrm{I}) \quad \lim _{n \rightarrow 0} \phi_{b}(b b)=\delta(b-0)
$$

In the present case our Bayesian estimator for $z(t)$ becomes

$$
\begin{aligned}
& z(n)=\frac{n}{n+\frac{E_{\theta} V(\xi / \theta)+\mathrm{I}}{V_{\theta} E(\xi / \theta)+n}}- \\
& \frac{e^{1 / 2 V_{\theta} E(\xi / \theta)+\frac{n}{2}-\frac{1}{2 n}\left[E_{\theta} V(\xi / \theta)+1\right]}}{\left[V_{\theta} E(\xi / \theta)+n+\frac{E_{\theta} V(\xi / \theta)+\mathrm{I}}{n}\right]} \times \int_{0}^{1} e^{-1 / 2 b^{2}\left[V_{\theta} E(\xi / \theta)+n\right]+n b V_{0} E(\xi / \theta)} d b
\end{aligned}
$$

However in our opinion the question arises whether it is necessary to have $\lim z(n)=\mathrm{I}$. Indeed, let us take a person who has a $n \rightarrow \infty$

zero past, it is $\sum_{t=1}^{n} x_{t}=0$. In that case his fair premium would be zero. It is clear that a priori $b$ has o and I as constraints, but it is not clear that $b$ necessary has to approach to one as $n \rightarrow \infty$. For our present $z(n)$ one has $\lim _{n \rightarrow \infty} z(n)=\mathrm{I}$.

In both cases one obtains:

$$
z(n)=\frac{n}{n+N}-C(n)
$$

But

$$
\begin{aligned}
E\left(\xi_{n+1} / \bar{x}\right) & =\left(\mathbf{I}-\frac{n}{n+N}\right) E(\xi)+\frac{n}{n+N} \frac{\mathrm{I}}{n} \sum_{t=1}^{n} x_{t} \\
& +C(n) \frac{\mathrm{I}}{n} \sum_{i=1}^{n} x_{t}-C(n) E(\xi)
\end{aligned}
$$


It is clear that

$$
E\left\{C(n) \frac{I}{n} \sum_{t=1}^{n} \xi_{t}-C(n) E(\xi)\right\}=0
$$

So that in fact the correction term can be omitted, no differences will occur in the collective. Although taking into account the correction term one avoids a priori zero premiums. Of course up to know we have introduced in our Bayesian estimation problem an a priori variance $\sigma=\mathrm{I}$, in fact $\sigma$ is unknown and has to be considered as a parameter. So we have to construct a new model.

\section{Estimation of the Credibility Factor in Case of Unknown}

\section{VARIANCE}

In fact one has to consider the problem of finding $b$ in the linear regression model

$$
E\left(\xi_{n+1} / \bar{x}\right)=E(\xi)+b\left(\frac{I}{n} \sum_{i=1}^{n} x_{t}-E(\xi)\right)+\varepsilon_{n}
$$

where $\varepsilon_{n}$ is an error term, supposed to be normaly distributed with mean value o and unknown dispersion $\sigma$. So the likelihood function becomes:

$$
L \cong \frac{\mathrm{I}}{\sigma^{N}} e^{-\frac{1}{2 \sigma^{2}}} \sum_{i=1}^{n} n_{i}\left(E\left(\xi_{n+1} \mid x_{1}{ }^{(i)}, \ldots, x_{n}{ }^{(i)}\right)-E(\xi)-b\left(\frac{\sum_{i=1}^{n} x_{i}{ }^{(i)}}{n}-E(\xi)\right)\right)^{2}
$$

Of course the $n_{i}$ will be proportional (statistically) to the probability for finding $x_{1}^{(i)} \ldots x_{n}^{(i)}$. Supposing the number of experiments large (it is the number of elements in the port folio is large) one has:

$$
\begin{array}{r}
\sum_{i=1}^{n} n_{i}\left(E\left(\xi_{n+1} / \bar{x}^{(i)}-E(\xi)-b\left(\frac{\mathrm{I}}{n} \sum_{t=1}^{n} x_{t}^{(i)}-E(\xi)\right)\right)^{2} \approx\right. \\
E\left[\left(E\left(\xi_{n+1} / x_{1} \ldots x_{n}\right)-E(\xi)-b\left(\frac{\mathrm{I}}{n} \sum_{t=1}^{n} \xi_{t}-E(\xi)\right)\right)^{2}\right]
\end{array}
$$

The proportionality factor is not important, because in performing the integration he cancels. 
Applying Jeffreys' rules one gets for the posterior probability density function:

$$
p d f(b) \cong \frac{I}{\sigma^{N+1}} e^{-\frac{1}{2 \sigma^{2}} E\left(\left(E\left(\xi_{n+1} / \bar{x}\right)-E(\xi)-b\left(1 / n \sum_{t=1}^{n} x_{t}-E(\xi)\right)\right)^{2}\right)}
$$

which can still be cast into the form

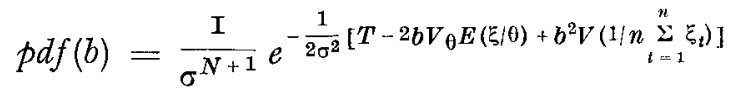

with:

$$
T=E\left[\left(E\left(\xi_{n+1} / \bar{\xi}\right)-E(\xi)\right)^{2}\right]
$$

Performing the integration over $\sigma$ gives:

$$
\begin{aligned}
p d f(b) \cong \frac{\mathrm{I}}{\left(T-2 b V_{\theta} E(\xi / \theta)+b^{2} V\left(\frac{\mathrm{I}}{n} \sum_{t=1}^{n} \xi_{t}\right)\right)^{N / 2}} \\
\cong \frac{\mathrm{I}}{\left(\mathrm{I}-2 b \frac{V_{\theta} E(\xi / \theta)}{T}-b^{2} \frac{V\left(\frac{\mathrm{I}}{n} \sum_{t=1}^{n} \xi_{t}\right)^{N / 2}}{T}\right)}
\end{aligned}
$$

Such that

$$
\text { B. E. }(b)=\frac{\int_{0}^{1} b d b\left[I-2 b \frac{V_{\theta} E(\xi / \theta)}{T}+b^{2}-\frac{V\left(\frac{I}{n} \sum_{t=1}^{n} \xi_{t}\right)}{T}\right]^{-N / 2}}{\int_{0}^{1} d b\left[I-2 b \frac{V_{\theta} E(\xi / \theta)}{T}+b^{2}-\frac{V\left(\frac{I}{n} \sum_{t=1}^{n} \xi_{t}\right)}{T}\right]^{-N / 2}}
$$

Introducing the new variable $b=(t / N)$ one gets:

B.E. $(b)=\frac{\int_{0}^{N} t d t\left(\mathrm{I}-2 t \frac{V_{\theta} E(\xi / \theta)}{N T}+t^{2} \frac{V\left(\frac{\mathrm{I}}{n} \sum_{t=1}^{n} \xi_{t}\right) / N}{N}\right)^{-N / 2}}{\int d t\left(\mathrm{I}-2 t \frac{V_{\theta} E(\xi / \theta)}{N T}+t^{2} \frac{V\left(\frac{\mathrm{I}}{n_{t-1}} \sum_{t=1}^{n} \xi_{t}\right) / N}{N}\right)^{-N / 2}}$ 
For large values of $N$ one gets:

$$
\text { B.E. }(b)=\frac{\int_{0}^{N} t d t e^{t \frac{V_{\theta} E(\xi / \theta)}{T}-1 / 2 t^{2} \frac{V\left(\frac{1}{n} \sum_{t-1}^{n} \xi_{t}\right) / N}{T}}}{\int_{0}^{N} d t e^{t \frac{V_{\theta} E(\xi / \theta)}{T}-1 / 2 t^{2}} \frac{V\left(\frac{1}{N} \sum_{t=1}^{n} \xi_{t}\right) / N}{T}}
$$

By means of one partial integration one gets:

$$
\begin{aligned}
& \text { B.E. }(b)=\frac{N T}{N_{4} V\left(\frac{I}{n} \sum_{t=1}^{n} \xi_{t}\right)} \\
& N \frac{V_{\theta} E(\xi ; \theta)}{T}-1 / 2 N \frac{V\left(\frac{1}{n} \sum_{t=1}^{n} \xi_{t}\right)}{T} \\
& \frac{e}{{ }_{N}} \frac{-\mathrm{I}}{t \frac{V_{\theta} E(\xi / \theta)}{T}-1 / 2 t^{2} V\left(\frac{1}{n} \sum_{t=1}^{n} \xi_{t}\right) N} \\
& \int_{0}^{\infty} d t e \\
& +\frac{T}{V\left(\frac{\mathrm{I}}{n} \sum_{t=1}^{n} \xi_{t}\right)} \cdot \frac{V_{\theta} E(\xi / \theta)}{T}
\end{aligned}
$$

And so, in the limit for $N$

$$
\text { B.E. }(b)=\frac{V_{\theta} E(\xi / \theta)}{V\left(\frac{I}{n} \sum_{t=1}^{n} \xi_{t}\right)}
$$

which gives Buhlmanns' result, having introduced as a prior condition that our estimation for $z(t)$ has to satisfy some inequality constraints.

\section{REFERENCES}

[I] VAn KLinken Dr. J. and Drs. G. W. Euverman, Overzicht van de theorie en toepassingen van credibility indices, (unpublished).

[2] Zellner, A. (I97I), An Introduction to Bayesian Inference in Econometrics, (eds) John Wiley and Sons, Inc., New York.

[3] Broeckx, F., Goovaerts, M. and J. van Den Broeck, On the prior density functions proposed by Jeffreys and Haldane, in a Bayesian framework, Sogesci, to be published. 
[4] Jewell, W. S., "The credible distribution", The ASTIN Bull., Vol. VII, Part 3, pp. 237-269.

[5] For other derivations of the credibility factor see references given by W. S. JEWELL in [4].

[6] BüHlmanv, H. (г970), Mathematical methods in risk theory, (eds) SpringerVerlag, New York.

[7] Bühlmann, H. (I971), Credibility Procedures, Sixth Berkeley Symposium, pp. 5I 5-525. 